\title{
Impacts of survivin and caspase-3 on apoptosis and angiogenesis in oral cancer
}

\author{
SHUXIA LI ${ }^{1}$, YANQI YANG ${ }^{2}$, YANPING DING ${ }^{1}$, XIAOFEI TANG ${ }^{3}$ and ZHENG SUN ${ }^{1}$ \\ ${ }^{1}$ Department of Oral Medicine, Beijing Stomatological Hospital of China, Capital Medical University, Beijing 100050; \\ ${ }^{2}$ Department of Orthodontics, School of Dentistry, Hong Kong University, Hong Kong 999077; \\ ${ }^{3}$ Institute of Stomatology, Capital Medical University, Beijing 100050, P.R. China
}

Received February 13, 2016; Accepted January 19, 2017

DOI: $10.3892 / 01.2017 .6626$

\begin{abstract}
The present study aimed to investigate the impact of survivin and caspase-3 on apoptosis and angiogenesis in oral cancer. A total of 16 oral leukoplakia cases accompanied by low-moderate epithelial dysplasia, 12 cases of oral leukoplakia accompanied by severe epithelial dysplasia, 17 cases of high-moderate differentiated oral squamous cell carcinoma and 10 cases of normal oral mucosa were selected. Immunohistochemistry was used to detect the expression levels of survivin, caspase-3, and caspase inhibitor factor VIII in lesions from each group. Terminal deoxynucleotidyl transferase 2'-deoxyuridine, 5'-triphosphate nick end labeling was performed to detect the apoptotic index of oral leukoplakia and cancer tissue. Immunohistochemistry revealed increased expression levels of survivin in oral cancer tissues, as compared with the normal mucosa, whereas the expression of Caspase-3 was decreased during malignant transformation. Microvascular density (MVD) was increased from $28.49 \pm 11.87$ strips $/ \mathrm{mm}^{2}$ (mean \pm standard deviation, normal control group) to $91.98 \pm 40.20$ strips $/ \mathrm{mm}^{2}$ (oral cancer group). Therefore, survivin may serve an important role in oral cancer, as its expression was increased in association with a downward trend in caspase-3 expression and apoptotic index, whereas MVD was significantly increased.
\end{abstract}

\section{Introduction}

An imbalance between cell proliferation and apoptosis is important during the occurrence and development of oral cancer. This dynamic balance is essential to maintaining homeostasis, which ensures the balance and stability of the human body at the cellular level. Previous studies have observed that the reduction of apoptosis also serves a key role in oral

Correspondence to: Professor Shuxia Li, Department of Oral Medicine, Beijing Stomatological Hospital of China, Capital Medical University, 4 Tiantan Xili, Dongcheng, Beijing 100050, P.R. China E-mail: lishuxiadc@126.com

Key words: caspase-3, oral cancer, apoptosis, angiogenesis cancer incidence and development. A previous study demonstrated that pro-apoptotic factors, including tumor protein 53 and Fas, as well as anti-apoptotic factors, including the B-cell lymphoma-2 family and inhibitors of apoptosis protein family (IAPs), serve crucial roles in the pathogenesis of oral cancer (1). Of the IAPs, survivin is the protein with the highest apoptosis inhibitory ability, and also regulates the cell cycle (2). Previous studies compared the expression profile of survivin in normal oral mucosa, oral precancerous lesions and oral cancer tissue, revealing that survivin was not expressed in the normal oral mucosa but was expressed in the early and precancerous stages of oral cancer (3-5). The positive expression rate of survivin in epithelial paraplastic tissues was $\sim 97$ and $\sim 98 \%$ in oral cancer tissue (3-5), and its expression in distinct splice variants was also altered during tumorigenesis (6). This indicates that survivin not only serves an important role in the occurrence of oral cancer, but that the increase in its expression levels are an early event in the development of this type of cancer. A previous study also observed that survivin was associated with angiogenesis, as it was not able not be detected in quiescent endothelial cells, but was strongly expressed in angiogenic factor-stimulated endothelial cells (7). In addition, the application of antisense survivin technology during angiogenesis resulted in the instant abrogation of the cytoprotective effects of vascular endothelial growth factor (VEGF), inhibition of blood vessel growth and apoptosis of endothelial cells (7). In the present study, immunohistochemistry and cell apoptosis detection were used to investigate the roles of survivin and caspase-3 (its downstream target in the apoptosis signaling pathway) in the incidence and development of oral cancer, and to explore the association between neovascularization and survivin expression during this process.

\section{Materials and methods}

Case selection. A total of 45 paraffin-embedded tissue specimens were obtained from the Department of Pathology, Beijing Stomatological Hospital (Beijing, China) from September 2005 to August 2007 were selected, including: 16 cases of oral leukoplakia accompanied by low-moderate epithelial dysplasia (OL-LMED) (age, 61.8 \pm 15.0 years old; 9 males and 7 females), 12 cases of oral leukoplakia accompanied by severe epithelial dysplasia (OL-SED) (age, 63.6 \pm 16.8 years 
old; 5 males and 7 females) and 17 cases of high-moderate differentiated oral squamous cell carcinoma (OSCC) (age, $62.4 \pm 14.0$ years old; 9 males and 8 females). The patients did not receive any treatments prior to enrolment in the present study. All tissue specimens were diagnosed by two experienced pathologists (Department of Pathology, Oral Medicine, Beijing Stomatological Hospital) according to the histologic classification criteria of oral mucosa cancer and precancerous lesions issued by the World Health Organization in 1996 (8). A further 10 normal oral mucosa tissue specimens were selected as controls. This study was conducted in accordance with the Declaration of Helsinki and with approval from the Ethics Committee of Capital Medical University (Beijing, China). Written informed consent was obtained from all participants.

Immunohistochemical streptavidin peroxidase assay. A 3,3'-diaminobenzidine chromogenic reagent kit (Fuzhou Maixin Biotech Co. Ltd., Fuzhou, China) was used to detect the expression of survivin (mouse anti-human survivin monoclonal antibody; dilution 1:400; catalogue number TA301427; OriGene Technologies, Inc., Rockville, MD, USA), caspase-3 (mouse anti-human caspase-3 monoclonal antibody; dilution 1:400; catalogue number 610322; BD Biosciences, Franklin Lakes, NJ, USA) and factor VIII (rabbit anti-human von Willebrand factor polyclonal antibody; dilution 1:400; catalogue number MBS1489331; MyBioSource, CA, USA) in the tissue samples. The incubation was performed at $4^{\circ} \mathrm{C}$ overnight. Goat anti-mouse $\operatorname{IgG}$ (dilution 1:500; catalogue number L3031; Signalway Antibody LLC, MA, USA) and goat anti-rabbit IgG (dilution 1:500; catalogue number L3041; Signalway Antibody) were used as the secondary antibodies. The incubation was performed at $37^{\circ} \mathrm{C}$ for $10 \mathrm{~min}$. Other experimental procedures were performed according to the manufacturer's protocols.

Terminal deoxynucleotidyl transferase 2'-deoxyuridine, 5'-triphosphate nick end labeling (TUNEL) assay. TUNEL was performed to detect apoptosis using the apoptosis detection kits (Wuhan Boster Biological Technology, Ltd., Wuhan, China), according to manufacturer's protocols. Double-distilled water was used to replace terminal deoxynucleotidyl transferase staining (negative control). The standard positive slice included in the kit was used as the positive control (Wuhan Boster Biological Technology, Ltd.). The reaction was terminated when the positive control achieved coloration, followed by observation with a light microscope and the quantification of TUNEL-positive cells.

Detection of survivin and caspase-3. Positive staining of survivin was primarily localized to the cytoplasm and nucleus, exhibiting uniform yellow-brown granules. Positive staining of caspase-3 was principally localized to the cytoplasm, or simultaneously expressed in the cytoplasm and the nucleus, also exhibiting uniform, yellow-brown granules. Five high power fields were counted (magnification, 400x), with a total cell count $>1,000$ in order to quantify the number of positive cells. According to the degree of staining, positive signals were subdivided into three categories, as follows: weakly positive (pale yellow, or individual cells were stained yellow-brown), moderately positive (intermediate staining) and intensely positive (dark brown staining.
Microvessel counting. Microvessel counting was performed using the Weidner method (9). Using this technique, the endothelial cells (identified by the positive staining of factor VIII) or cell clusters that exhibited clear boundaries between adjacent microvessels and cancerous cells in tumor tissues, and stained brown or tan were considered as the nascent tumor blood vessel. The appearance of red blood cells or lumen was not included in the judgment criteria, and for blood vessels with a thick muscular layer and a lumen diameter $>8$, red blood cells were not counted. For each lesion, $\geq 3$ fields were randomly selected (magnification, 200x) to determine the number of blood vessels, and image analysis software (ImagePro Plus version 6; Media Cybernetics Inc., Rockville, MD, USA) was used to calculate the number of microvessels (strips $/ \mathrm{mm}^{2}$ ) per unit area. The mean value of three fields of vision was used as the final microvascular density (MVD) for each patient tissue sample.

Detection of apoptosis. A cell with brown granules in the nucleus was considered to be positive and, therefore, an apoptotic cell. For each tissue section, the five most positively stained high-power fields (magnification, 400x) were randomly selected to count $>500$ cells and calculate the apoptotic index (AI), which was defined as the numbers of apoptotic cells per 100 cells.

Statistical analysis. Data were presented as the mean \pm standard deviation. Comparisons of survivin expression and caspase-3 expression, and the associations between AI and tissue type were analyzed using the non-parametric Kruskal-Wallis test. Fisher's least significant difference method was used for intergroup comparison. The immunohistochemical staining of factor VIII was analyzed using SPSS statistical software version 11.5 for Windows (SPSS, Inc., Chicago, IL, USA). Analysis of variance was used to analyze normally distributed data, whilst non-normal distributions were analyzed using the rank sum test. Intergroup paired comparison was then performed to examine any statistically significant differences. $\mathrm{P}<0.05$ was considered to indicate a statistically significant difference.

\section{Results}

Immunohistochemical assay. Survivin was primarily expressed in the cytoplasm, nucleus and membrane of the epithelial cells, as well as in the cytoplasm of inflammatory stromal cells. The majority of normal tissues exhibited no significant survivin expression; however, in accordance with the progression from epithelial dysplasia to oral cancer the expression levels of survivin gradually increased (Fig. 1), and the expression levels in the tumor edge were greater, as compared with those in the tumor center. Staining of survivin exhibited heterogeneity, and the staining intensity and staining area varied between tissues and regions. The expression of survivin in the OSCC group was significantly increased compared with the OL-LMED, OL-SED and normal control group $(\mathrm{P}<0.05$; Table I).

Caspase-3 positive staining was observed as brown particles, primarily either in the cytoplasm of epithelial cells or in the nucleus, and rarely on the nuclear membrane. The normal 
Table I. Expression of survivin in various groups (mean \pm standard deviation).

\begin{tabular}{lcc}
\hline Group & Cases, $\mathrm{n}$ & $\begin{array}{c}\text { Average proportion } \\
\text { of positive cells, \% }\end{array}$ \\
\hline Normal control & 10 & $1.05 \pm 1.21^{\mathrm{a}}$ \\
LMED & 16 & $6.06 \pm 4.87^{\mathrm{a}}$ \\
SED & 12 & $12.49 \pm 8.41^{\mathrm{a}}$ \\
OSCC & 17 & $21.89 \pm 10.45$ \\
\hline
\end{tabular}

${ }^{\text {a }}<0.05$ compared with the OSCC group. LMED, low-moderate epithelial dysplasia; SED, severe epithelial dysplasia; OSCC, oral squamous cell carcinoma.

control group exhibited positive caspase-3 expression (10/10), with the majority exhibiting intense positive staining (Fig. 2). Corresponding to the progression from epithelial dysplasia to oral cancer, the expression levels of caspase- 3 were gradually decreased. Immunostaining exhibited heterogeneity and the staining intensity and staining area changed in various tissues and regions. The expression of caspase- 3 in the normal control group, OL-LMED group and OL-SED group were significantly increased compared with in the OSCC group $(\mathrm{P}<0.05$; Table II).

MVD in the normal control group, OL-LMED group, OL-SED group and OSCC group was $28.49 \pm 11.87$, $47.92 \pm 25.58,49.73 \pm 23.76$ and $91.98 \pm 40.20$ strips $/ \mathrm{mm}^{2}$, respectively. A gradual increase in MVD was observed from the normal control group to the OL-LMED group, then the OL-SED group, with the highest MVD in the OSCC group (Table III). Comparisons between the normal control group and OSCC group, between the OL-LMED group and OSCC group, and between the OL-SED group and OSCC group all exhibited statistically significant differences $(\mathrm{P}<0.05$; Fig. 3).

AI. Apoptotic cells were stained brownish yellow using TUNEL and exhibited pyknosis. Cells were round and oval, and a number were plate-shaped or crescent-shaped. The majority of apoptotic cells were scattered and had no surrounding inflammation. The AI of the normal control group, OL-LMED group, OL-SED group and OSCC group was $0.89 \pm 0.46,1.29 \pm 0.63$, $0.65 \pm 0.40$ and $0.21 \pm 0.12$, respectively (mean \pm standard deviation; Fig. 4). The AI was significantly decreased in the OSCC group, with apoptotic cells predominantly distributed around the tumor core and rarely observed in the tumor frontier. AI in normal control group, OL-LMED group and OL-SED group were significantly higher compared with in the OSCC group $(\mathrm{P}<0.05$; Table IV).

\section{Discussion}

The occurrence and development of oral cancer is a multi-stage process regulated by numerous genes, and includes the accumulation of mutations and imbalances in accumulation and cell cycle regulation (10). These changes may be able to be detected in the precancerous lesion stage, potentially aiding early prediction and prevention of oral cancer.
Table II. Expressions of caspase-3 in various groups (mean \pm standard deviation).

\begin{tabular}{lcc}
\hline Group & Cases, $\mathrm{n}$ & $\begin{array}{c}\text { Average proportion of } \\
\text { positive cells, \% }\end{array}$ \\
\hline Normal control & 10 & $12.37 \pm 5.48^{\mathrm{a}}$ \\
LMED & 16 & $19.51 \pm 13.15^{\mathrm{a}}$ \\
SED & 12 & $9.76 \pm 7.83^{\mathrm{a}}$ \\
OSCC & 17 & $6.08 \pm 6.91$ \\
\hline
\end{tabular}

${ }^{\text {aP }}<0.05$ compared with the OSCC group. LMED, low-moderate epithelial dysplasia; SED, severe epithelial dysplasia; OSCC, oral squamous cell carcinoma.

Table III. MVD in different groups (mean \pm standard deviation).

\begin{tabular}{lcc}
\hline Group & Cases, $\mathrm{n}$ & MVD, strips $/ \mathrm{mm}^{2}$ \\
\hline Normal control & 10 & $28.49 \pm 11.87^{\mathrm{a}}$ \\
LMED & 16 & $47.92 \pm 25.58^{\mathrm{a}}$ \\
SED & 12 & $49.73 \pm 23.76^{\mathrm{a}}$ \\
OSCC & 17 & $94.97 \pm 40.32$ \\
\hline
\end{tabular}

${ }^{\mathrm{a}} \mathrm{P}<0.05$ compared with the OSCC group. MVD, microvascular density; LMED, low-moderate epithelial dysplasia; SED, severe epithelial dysplasia; OSCC, oral squamous cell carcinoma.

Table IV. AI in various groups (mean \pm standard deviation)

\begin{tabular}{lcc}
\hline Group & Cases, $\mathrm{n}$ & AI, \% \\
\hline Normal control & 10 & $0.89 \pm 0.46^{\mathrm{a}}$ \\
LMED & 16 & $1.29 \pm 0.63^{\mathrm{a}, \mathrm{b}}$ \\
SED & 12 & $0.65 \pm 0.40^{\mathrm{a}}$ \\
OSCC & 17 & $0.21 \pm 0.12$ \\
\hline
\end{tabular}

${ }^{\mathrm{a}} \mathrm{P}<0.05$ compared with the OSCC group, ${ }^{\mathrm{b}} \mathrm{P}<0.05$ compared with the SED group. AI, apoptotic index; LMED, low-moderate epithelial dysplasia; SED, severe epithelial dysplasia; OSCC, oral squamous cell carcinoma.

Survivin is considered to be the most potent apoptosis inhibitor ever discovered. A previous study demonstrated that no, or low, expression of survivin is able to be observed in normal tissues, whereas this protein it is specifically and highly expressed in tumor tissues, and this expression is tumor cell-dependent (11). In the present study, survivin was also specifically expressed in tumor cells of the oral mucosa. Previous studies used immunohistochemical assays to detect the expression of survivin in oral mucosae, and observed that its expression in precancerous lesions that had not yet progressed towards malignancy and had progressed to complete squamous cancer were 33\% (10/30) and 94\% (15/16), respectively $(12,13)$. 

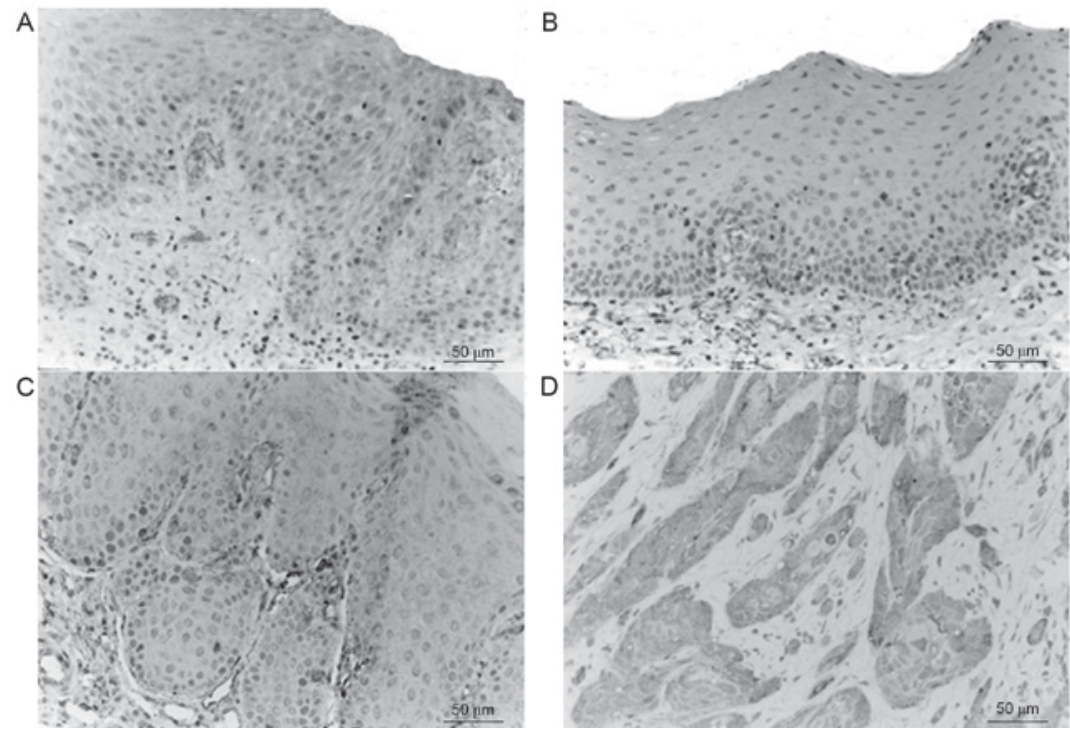

Figure 1. Expressions of Survivin in various groups. (A) Normal oral mucosa tissue; (B) oral leukoplakia accompanied by low-moderate epithelial dysplasia; (C) oral leukoplakia accompanied by severe epithelial dysplasia; (D) high-moderate differentiated oral squamous cell carcinoma.
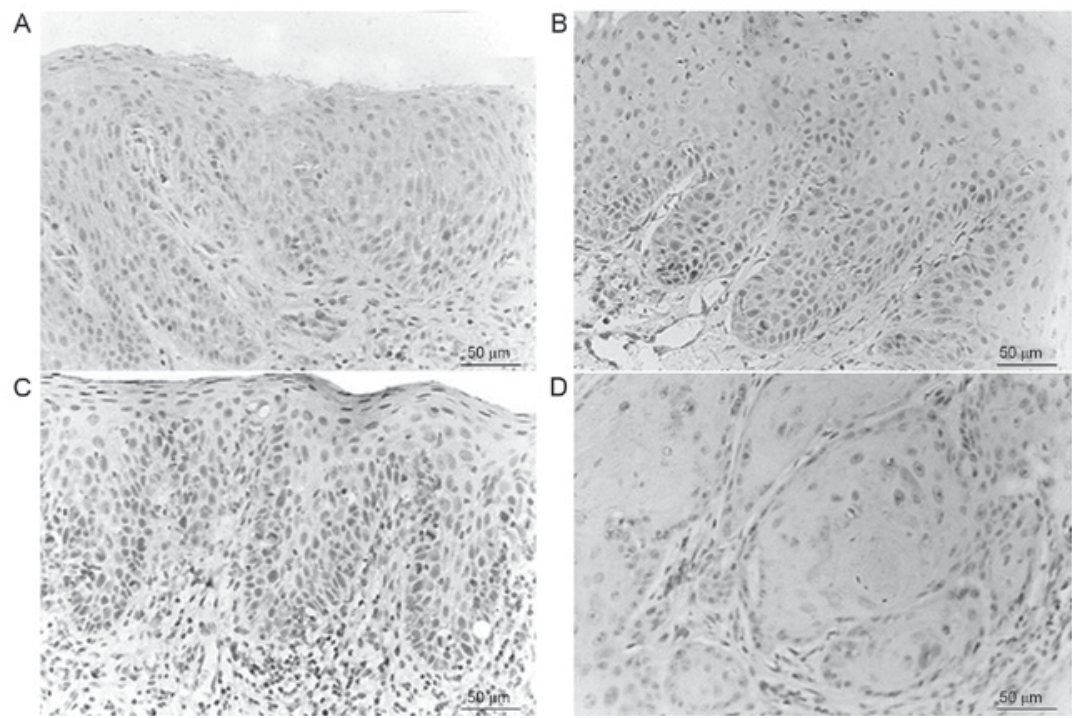

Figure 2. Expressions of Caspase-3 in various groups. (A) Normal oral mucosa tissue; (B) oral leukoplakia accompanied by low-moderate epithelial dysplasia; (C) oral leukoplakia accompanied by severe epithelial dysplasia; (D) high-moderate differentiated oral squamous cell carcinoma.

Previous studies revealed that survivin gene expression was also present in various precancerous damaged tissues, including oral leukoplakia, colon polyps, mastitis or keratotic dermatitis $(14,15)$. The present study obtained similar results, as the expression levels of survivin were gradually increased during the progression from abnormal cell proliferation to malignant transformation.

Caspase- 3 is expressed in normal tissues and its expression level in gastric cancer is significantly reduced, which is negatively correlated with survivin expression level (16). Previous studies detected survivin and caspase- 3 mRNA expression in tongue cancer, demonstrating that the overexpressed survivin mRNA inhibited the expression of caspase-3 mRNA $(17,18)$. The expression of the two were negatively correlated and associated with the occurrence and progression of tumors $(17,18)$. The results of the present study were concordant with these findings, in that as survivin expression levels increased, the expression levels of caspase-3 gradually decreased.

Survivin was specifically highly expressed during tumorigenesis, particularly in early-stage cancer, and its expression was regulated by a variety of factors $(19,20)$. Therefore, it is possible that during the formation of oral cancer, a variety of oncogene signaling pathways may induce increased expression levels of survivin in tumor tissues.

Furthermore, the AI in the OL-LMED group was increased from that observed in normal oral tissues, which may occur as part of the tissue stress response towards the lesions. In oral precancerous lesions, the cells exhibited rapid growth, while apoptosis was increased in order to balance the resulting proliferation-apoptosis disorder. Furthermore, during the carcinogenesis of the oral mucosa the underlying mechanisms 

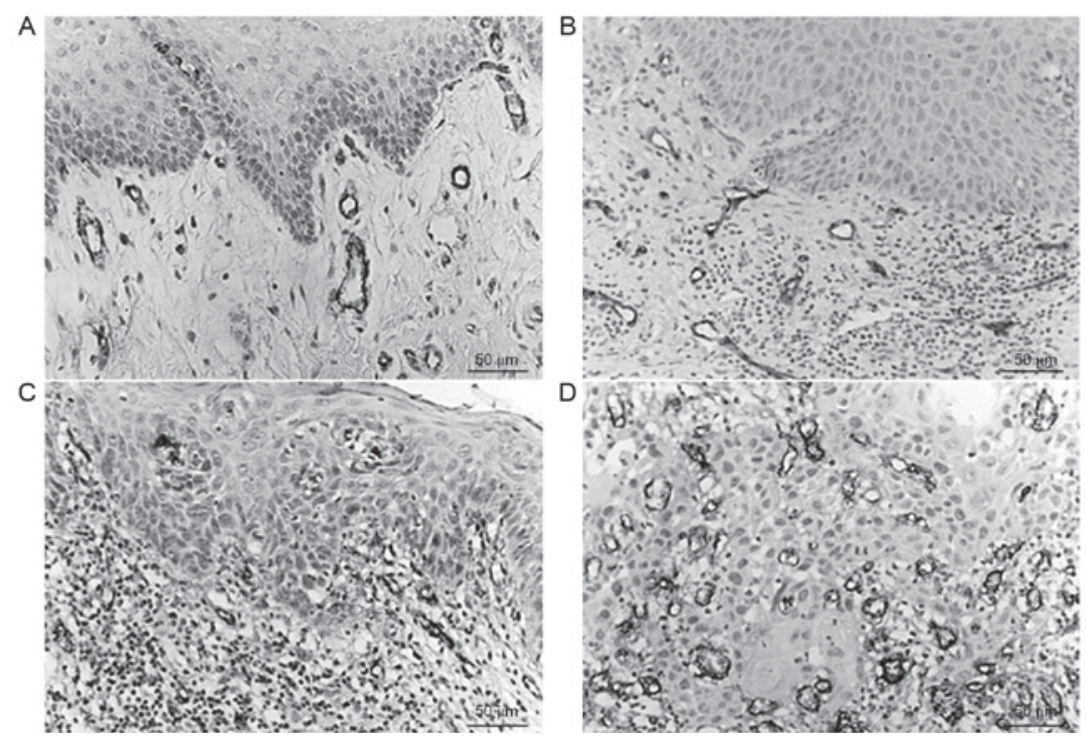

Figure 3. Expressions of VIII factor in various groups. (A) Normal oral mucosa tissue; (B) oral leukoplakia accompanied by low-moderate epithelial dysplasia; (C) oral leukoplakia accompanied by severe epithelial dysplasia; (D) high-moderate differentiated oral squamous cell carcinoma.
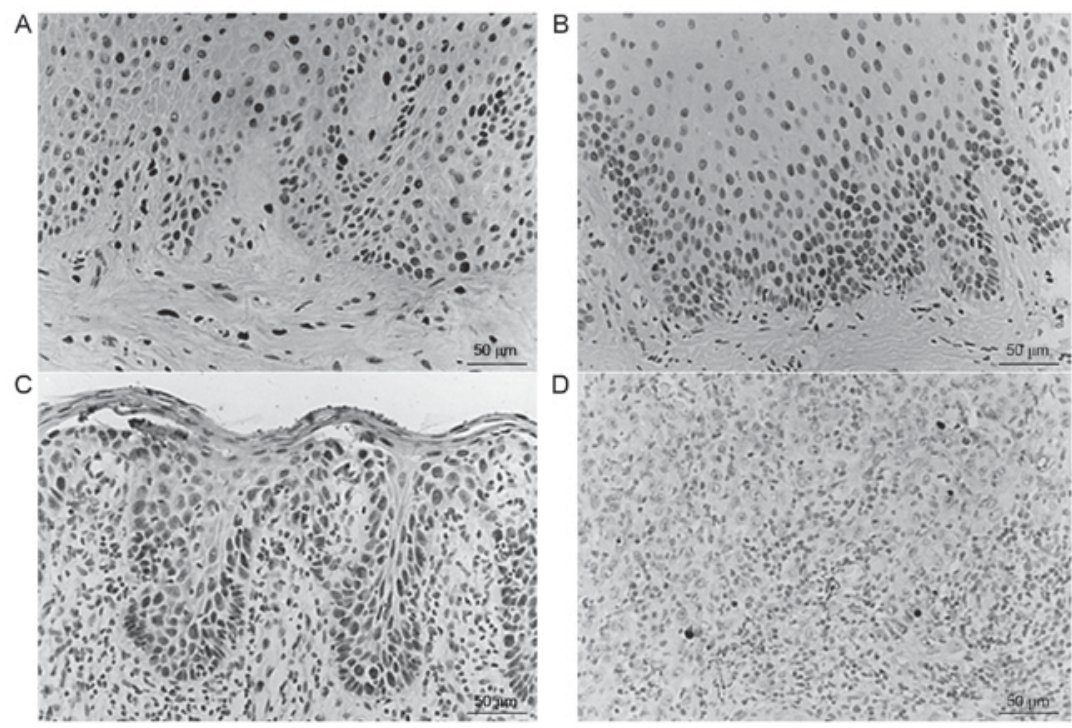

Figure 4. Expressions of apoptotic cells in various groups. (A) Normal oral mucosa tissue; (B) oral leukoplakia accompanied by low-moderate epithelial dysplasia; (C) oral leukoplakia accompanied by severe epithelial dysplasia; (D) high-moderate differentiated oral squamous cell carcinoma.

of apoptosis, including p53 mutations and the downregulation of caspase-3, were inhibited. The inhibition of apoptosis simultaneously promoted the occurrence of severe epithelial dysplasia and canceration, concordant with the results of previous studies $(21,22)$.

Of the angiogenesis-promoting factors, VEGF is highly expressed in the majority of human and animal tumors. VEGF may significantly increase the expression levels of survivin, which may then inhibit the caspase-mediated apoptosis signaling pathway, thus protecting the nascent immature tumor vascular endothelial cells, allowing them to evade apoptosis and subsequently produce more VEGF $(23,24)$. It was hypothesized that highly expressed survivin may promote uncontrolled cell cycle progression, bypassing the $\mathrm{G}_{2} / \mathrm{M}$ DNA damage checkpoint. Therefore, the cells may lose the restriction of the apoptosis switch during normal cell proliferation, inducing malignant transformation and a proliferation-apoptosis imbalance in cells. Survivin may also promote the proliferation of endothelial cells, and silencing survivin may be able to inhibit the expression of VEGF (25). The import of survivin-targeting antisense oligonucleotides into cultured endothelial cells may promote the apoptosis of these endothelial cells and inhibit angiogenesis $(26,27)$. A previous study has demonstrated that basic fibroblast growth factor and survivin also produce synergistic effects, with positively associated expression patterns in lung cancer and OSCC (23). This may promote the proliferation of tumor and endothelial cells, and enhance angiogenesis (28).

Through analyzing the expression profile of survivin and MVD in oral leukoplakia and oral cancer tissues, similar conclusions may be obtained. These are namely that, corresponding with the increased expression levels of survivin in oral cancer tissues, MVD was also increased and exhibited a 
marked increasing trend, indicating that survivin was involved in tumor angiogenesis, which may serve an important role in the occurrence and development of oral cancer.

In conclusion, the present study demonstrated that survivin and caspase- 3 served important roles in the occurrence and development of oral cancer and, therefore, may be of potential use in monitoring the progression of oral cancer. As survivin was differentially expressed in tumor tissues and normal tissues, highly expressed in tumor cells and the vascular endothelium and angiogenesis was critically dependent on the viability of the endothelial cells, any survivin-targeting treatment may be able to promote the apoptosis and degeneration of the vessels, and indirectly inhibit tumor growth. Therefore, survivin has the potential to become a novel therapeutic target for certain types of cancer. Further in-depth understanding regarding the structures, properties and physiological effects of survivin and caspase-3 may facilitate the full elucidation of their roles in apoptosis, therefore providing more options for the prevention of cancer, and for the development of novel agents to target tumors and numerous other chronic diseases. It is speculated that this approach may provide new treatments, with reduced adverse effects on normal tissues, whilst effectively inhibiting the proliferation of tumor cells and tumor growth.

\section{Acknowledgments}

The present study was supported by grants from the National Natural Science Foundation of China (grant no. 30500559), Postdoctoral Science Foundation of China (grant no. 20060400486) and The New Star Projects of Beijing Municipal Science and Technology (grant no. 2005B51).

\section{References}

1. Sugerman PB, Joseph BK and Savage NW: Review article: The role of oncogenes, tumour suppressor genes and growth factors in oral squamous cell carcinoma: A case of apoptosis versus proliferation. Oral Dis 1: 172-188, 1995.

2. Nassar A, Lawson D, Cotsonis G and Cohen C: Survivin and caspase-3 expression in breast cancer: Correlation with prognostic parameters, proliferation, angiogenesis, and outcome. Appl Immunohistochem Mol Morphol 16: 113-120, 2008.

3. Lin CY, Hung HC, Kuo RC, Chiang CP and Kuo MY: Survivin expression predicts poorer prognosis in patients with areca quid chewing-related oral squamous cell carcinoma in Taiwan. Oral Oncol 41: 645-654, 2005.

4. Lauxen IS, Oliverira MG, Rados PV, Lingen MW, Nör JE and Sant'ana Filho M: Immunoprofiling of oral squamous cell carcinomas reveals high p63 and survivin expression. Oral Dis 20: e76-e80, 2014

5. Cai Y, Ma W, Huang X, Cao L, Li H, Jiang Y, Lu N and Yin Y: Effect of survivin on tumor growth of colorectal cancer in vivo. Int J Clin Exp Pathol 8: 13267-13272, 2015.

6. Mishra R, Palve V, Kannan S, Pawar S and Teni T: High expression of survivin and its splice variants survivin $\triangle \mathrm{Ex} 3$ and survivin 2 B in oral cancers. Oral Surg Oral Med Oral Pathol Oral Radiol 120: 497-507, 2015.

7. Altieri DC: The molecular basis and potential role of survivin in cancer diagnosis and therapy. Trends Mol Med 7: 542-547, 2001.

8. Axéll T, Pindborg JJ, Smith CJ and van der Waal I: Oral white lesions with special reference to precancerous and tobaccorelated lesions: Conclusions of an international symposium held in Uppsala, Sweden, May 18-21 1994. International collaborative group on oral white lesions. J Oral Pathol Med 25: 49-54, 1996.
9. Deng J, Kloosterbooer F, Xia W and Hung MC: The $\mathrm{NH}(2)$-terminal and conserved region 2 domains of adenovirus E1A mediate two distinct mechanisms of tumor suppression. Cancer Res 62: 346-350, 2002.

10. Rowley H, Sherrington P, Helliwell TR, Kinsella A and Jones AS: p53 expression and p53 gene mutation in oral cancer and dysplasia. Otolaryngol Head Neck Surg 118: 115-123, 1998.

11. Duffy MJ, O'Donovan N, Brennan DJ, Gallagher WM and Ryan BM: Survivin: A promising tumor biomarker. Cancer Lett 249: 49-60, 2007.

12. Lo Muzio L, Pannone G, Leonardi R, Staibano S, Mignogna MD, De Rosa G, Kudo Y, Takata T and Altieri DC: Survivin, a potential early predictor of tumor progression in the oral mucosa. J Dent Res 82: 923-928, 2003.

13. Poomsawat S, Punyasingh J and Vejchapipat P: Overexpression of survivin and caspase 3 in oral carcinogenesis. Appl Immunohistochem Mol Morphol 22: 65-71, 2014.

14. Grossman D, McNiff JM, Li F and Altieri DC: Expression of the apoptosis inhibitor, survivin, in nonmelanoma skin cancer and gene targeting in a keratinoyte cell line. J Lab Invest 79: 1121-1126, 1999.

15. Li SX, Chai L, Cai ZG, Jin LJ, Chen Y, Wu HR and Sun Z: Expression of survivin and caspase 3 in oral squamous cell carcinoma and peritumoral tissue. Asian Pac J Cancer Prev 13: 5027-5031, 2012 .

16. Kania J, Konturek SJ, Marlicz K, Hahn EG and Konturek PC: Expression of survivin and caspase-3 in gastric cancer. Dig Dis Sci 48: 266-271, 2003.

17. Wang ZM, Zhao ZG, Guan XM, Liu F and Zhang LP: Expression of Survivin mRNA and Caspase-3 mRNA and their correlation in tongue squamous cell carcinoma. Shanghai Kou Qiang Yi Xue 16: 582-586, 2007 (In Chinese)

18. Xu JH, Wang AX, Huang HZ, Wang JG, Pan CB and Zhang B: Survivin shRNA induces caspase-3-dependent apoptosis and enhances cisplatin sensitivity in squamous cell carcinoma of the tongue. Oncol Res 18: 377-385, 2010.

19. Holdenrieder S and Stieber P: Circulating apoptotic markers in the management of non-small cell lung cancer. Cancer Biomark 6: 197-210, 2010

20. Zhang JY, Casiano CA, Peng XX, Koziol JA, Chan EK and Tan EM: Enhancement of antibody detection in cancer using panel of recombinant tumor-associated antigens. Cancer Epidemiol Biomarkers Prev 12: 136-143, 2003.

21. Srinivasprasad V, Dineshshankar J, Sathiyajeeva J, Karthikeyan M, Sunitha J and Ragunathan R: Liaison between micro-organisms and oral cancer. J Pharm Bioallied Sci 7 (Suppl 2): S354-S360, 2015.

22. Hills SA and Diffley JF: DNA replication and oncogene-induced replicative stress. Curr Biol 24: R435-R444, 2014.

23. Wang $\mathrm{S}, \mathrm{Xu} \mathrm{J}$ and Zhang Q: Clinical significance of survivin and vascular endothelial growth factor mRNA detection in the peripheral whole blood of breast cancer patients. Neoplasma 63: 133-140, 2016.

24. Gao J, Huang Y, Li M, Zhao H, Zhao Y, Li R, Yan J, Yu Y and Qiao J: Effect of local basic fibroblast growth factor and vascular endothelial growth factor on subcutaneously allotransplanted ovarian tissue in ovariectomized mice. PLoS One 10: e0134035, 2015.

25. Ji ZJ, Wang JL and Chen L: Inhibition of skin squamous cell carcinoma proliferation and promote apoptosis by dual silencing of NET-1 and survivin. Oncol Rep 34: 811-822, 2015.

26. Coma S, Noe V, Lavarino C, Adán J, Rivas M, López-Matas M, Pagan R, Mitjans F, Vilaró S, Piulats J and Ciudad CJ: Use of siRNAs an d antisense oligonucleotides against survivin RNA to inhibit steps leading to tumor angiogenesis. Oligonucleotides 14: 100-113, 2004.

27. Tu SP, Cui JT, Liston P, Huajiang X, Xu R, Lin MC, Zhu YB, Zou B, Ng SS, Jiang SH, et al: Gene therapy for colon cancer by adeno-associated viral vector-mediated transfer of surviving Cys84Ala mutant. Gastroenterology 128: 361-375, 2005.

28. Cosgrave N, Hill AD and Young LS: Growth factor-dependent regulation of survivin by c-myc in human breast cancer. $\mathrm{J}$ Mol Endocrinol 37: 377-390, 2006. 